Commun. Fac. Sci. Univ. Ank. Ser. A1 Math. Stat.

Volume 69, Number 1, Pages 37-48 (2020)

DOI: $10.31801 /$ cfsuasmas. 484437

ISSN 1303-5991 E-ISSN 2618-6470

http://communications.science.ankara.edu.tr/index.php?series=A1

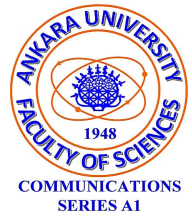

\title{
STUDY OF A GENERALIZED RIEMANN-LIOUVILLE FRACTIONAL INTEGRAL VIA CONVEX FUNCTIONS
}

\author{
GHULAM FARID
}

\begin{abstract}
In this paper estimations in general form of sum of left and right sided Riemann-Liouville (RL) fractional integrals for convex functions are studied. Also some similar fractional inequalities for functions whose derivatives in absolute value are convex, have been obtained. Associated fractional integral inequalities provide the bounds of different known fractional inequalities. These results may be useful in in the study of uniqueness solutions of fractional differential equations and fractional boundary value problems.
\end{abstract}

\section{INTRODUCTION}

Fractional calculus is applied in almost all disciplines of engineering and modern sciences. Since nineteenth century it has been acknowledged significantly and several new directions and subjects are invented. For example fractional geometry, fractional differential equations and fractional dynamics are due to fractional calculus.

Fractional integral operators play a leading and keen role in the development of fractional calculus. A first formulation of a fractional integral operator is due to a continuous study of well renowned mathematicians and physicist. This fractional integral is well known as Riemann-Liouville (RL) fractional integral operator. After its existence there have been introduced many other fractional integral and fractional derivative operators.

Now a days scientists in their diverse fields are working in the environment of fractional calculus and new directions of respective fields are developing rapidly. Theory of convex functions is the subject of mathematics that connects the mathematical analysis with other branches of science and engineering. Convex functions play an important role in the advancement of optimization theory, majorization theory, probability and statistics. A real valued function $f:[a, b] \rightarrow \mathbb{R}$ is said to

Received by the editors: November 16, 2018; Accepted: July 09, 2019.

2010 Mathematics Subject Classification. Primary 26A51; Secondary 26A33, 26D15.

Key words and phrases. Convex function, fractional integrals, bounds.

(C)2020 Ankara University Communications Faculty of Sciences University of Ankara-Series A1 Mathematics and Statistics 
be convex if $f(t x+(1-t) y) \leq t f(x)+(1-t) f(y)$ holds for all $x, y \in[a, b]$ and $t \in[0,1]$. If $-f$ is convex, then $f$ is said to be concave on $[a, b]$.

Convex functions are very close to the theory of inequalities. Many known and useful inequalities are consequences of convex functions. Some very natural inequalities for example Jensen inequality, Hadamard inequality interpret convex functions beautifully. Fractional integral inequalities occur by default in the study of convex and related functions due to applications of definitions of fractional integral as well as fractional derivative operators. Fractional integral inequalities are very useful in the study of fractional partial as well as ordinary differential equations. These are used to establish the uniqueness and bounds of their solutions. For detailed study suggested references are [15, 13, 9, 14, 11, 12, 17.

In this paper we study a general form of Riemann-Liouville (RL) fractional integrals via convex functions. Therefore it is need to give definitions of used fractional integrals. We start with the definition of Riemann-Liouville (RL) fractional integral.

Definition 1. Let $f \in L[a, b]$. Then the left-sided and right-sided Riemann Liouville fractional integrals of order $\alpha>0$ with $a \geq 0$ are defined as:

$$
\begin{aligned}
& I_{a+}^{\alpha} f(x)=\frac{1}{\Gamma(\alpha)} \int_{a}^{x}(x-t)^{\alpha-1} f(t) d t, \quad x>a \\
& I_{b-}^{\alpha} f(x)=\frac{1}{\Gamma(\alpha)} \int_{x}^{b}(t-x)^{\alpha-1} f(t) d t, \quad x<b,
\end{aligned}
$$

where $\Gamma$ (.) is the Gamma function.

A slight generalization of $(\mathrm{RL})$ fractional integral is Riemann-Liouville $(k-\mathrm{RL})$ $k$-fractional integral (see, [5]).

Definition 2. Let $f \in L[a, b]$. Then the $k$-fractional integrals of order $\alpha, k>0$ with $a \geq 0$ are defined as:

$$
\begin{aligned}
& I_{a+}^{\alpha, k} f(x)=\frac{1}{k \Gamma_{k}(\alpha)} \int_{a}^{x}(x-t)^{\frac{\alpha}{k}-1} f(t) d t, \quad x>a \\
& I_{b-}^{\alpha, k} f(x)=\frac{1}{k \Gamma_{k}(\alpha)} \int_{x}^{b}(t-x)^{\frac{\alpha}{k}-1} f(t) d t, \quad x<b,
\end{aligned}
$$

where $\Gamma_{k}($.$) is the k$-Gamma function (see, [2]).

A more general definition of (RL) fractional integral is the Riemann-Liouville fractional integral with respect an increasing function (see, [9]).

Definition 3. Let $f:[a, b] \rightarrow \mathbb{R}$ be an integrable function. Also let $g$ be an increasing and positive function on $(a, b]$, having a continuous derivative $g^{\prime}$ on $(a, b)$. 
The left-sided and right-sided fractional integrals of a function $f$ with respect to another function $g$ on $[a, b]$ of order $\alpha>0$ are defined as:

$$
\begin{gathered}
I_{g, a^{+}}^{\alpha} f(x)=\frac{1}{\Gamma(\alpha)} \int_{a}^{x}(g(x)-g(t))^{\alpha-1} g^{\prime}(t) f(t) d t, x>a \\
I_{g, b_{-}}^{\alpha} f(x)=\frac{1}{\Gamma(\alpha)} \int_{x}^{b}(g(t)-g(x))^{\alpha-1} g^{\prime}(t) f(t) d t, x<b .
\end{gathered}
$$

A $k$-analogue of above definition is defined in the next definition [10].

Definition 4. Let $f:[a, b] \rightarrow \mathbb{R}$ be an integrable function. Also let $g$ be an increasing and positive function on $(a, b]$, having a continuous derivative $g^{\prime}$ on $(a, b)$. The left-sided and right-sided fractional integrals of a function $f$ with respect to another function $g$ on $[a, b]$ of order $\alpha, k>0$ are defined as:

$$
\begin{aligned}
& I_{g, a^{+}}^{\alpha, k} f(x)=\frac{1}{k \Gamma_{k}(\alpha)} \int_{a}^{x}(g(x)-g(t))^{\frac{\alpha}{k}-1} g^{\prime}(t) f(t) d t, x>a \\
& I_{g, b-}^{\alpha, k} f(x)=\frac{1}{k \Gamma_{k}(\alpha)} \int_{x}^{b}(g(t)-g(x))^{\frac{\alpha}{k}-1} g^{\prime}(t) f(t) d t, \quad x<b .
\end{aligned}
$$

This is a compact form of a several fractional integral operators which are independently defined by the researchers in recent decade. The following lemma comprises on the formation of some particular fractional integrals which one can obtain from (3) and (4).

Remark 5. In the above Definition 4

(i) If we take $k=1$, then we get the Definition 3 of Riemann-Liouville fractional integrals with respect to an increasing function.

(ii) If we take $g(x)=x$, then we get the Definition 2 of Riemann-Liouville $k$ fractional integrals.

(iii) If we take $g(x)=x$ and $k=1$, then we get the Definition 1 of RiemannLiouville fractional integrals.

(iv) If we take $g(x)=\frac{x^{\rho}}{\rho}, \rho>0$ and $k=1$, then we get the definition of Katugampola fractional integrals given in [1].

(v) If we take $g(x)=\frac{x^{\tau+s}}{\tau+s}$ and $k=1$, then we get the definition of generalized conformable fractional integrals defined by T. U. Khan et al. in 8 .

(vi) If we take $g(x)=\frac{(x-a)^{s}}{s}, s>0$ in $\left.\sqrt{3}\right)$ and $g(x)=-\frac{(b-x)^{s}}{s}, s>0$ in (4), then we get the definition of conformable $(k, s)$-fractional integrals defined by Habib et al. in [6].

(vii) If we take $g(x)=\frac{x^{1+s}}{1+s}$, then we get the definition of generalized conformable fractional integrals defined by Sarikaya et al. in [16].

(viii) If we take $g(x)=\frac{(x-a)^{s}}{s}, s>0$ in 3 ) and $g(x)=-\frac{(b-x)^{s}}{s}, s>0$ in (4) with $k=1$, then we get the definition of conformable fractional integrals defined by $F$. Jarad et al. in 7 . 
The paper is organized as follows:

In Section 2, bounds of sum of the left and right sided (RL) $k$-fractional integrals in general form defined in Definition 4 have been established. Some related similar results are also obtained. These results are achieved by means of monotonicity and convexity properties. The presented results are useful in the study of fractional differential equations and fractional boundary value problems. Also they provide the estimations of Riemann-Liouville fractional integrals which are published in [4] and some results of [3. In Section 3 applications are discussed.

\section{Main Results}

The first result provides the estimates of sum of the left and right sided general (RL) fractional integral defined in Definition 4. Convexity and monotonicity properties of real valued functions are used.

Theorem 6. Let $f, g:[a, b] \longrightarrow \mathbb{R}$ be real valued functions with $a<b$. Also let $f$ be positive convex, and $g$ be differentiable and strictly increasing function with $g^{\prime} \in L[a, b]$. Then for $\alpha, \beta \geq k$, the following estimate is valid

$$
\begin{aligned}
& k \Gamma_{k}(\alpha) I_{g, a^{+}}^{\alpha, k} f(x)+k \Gamma_{k}(\beta) I_{g, b^{-}}^{\beta, k} f(x) \\
& \leq \frac{(g(x)-g(a))^{\frac{\alpha}{k}-1}}{x-a}\left[(x-a)(f(x) g(x)-f(a) g(a))-(f(x)-f(a)) \int_{a}^{x} g(t) d t\right] \\
& +\frac{(g(b)-g(x))^{\frac{\beta}{k}-1}}{b-x}\left[(b-x)(f(b) g(b)-f(x) g(x))-(f(b)-f(x)) \int_{x}^{b} g(t) d t\right] .
\end{aligned}
$$

Proof. Since the function $g$ is differentiable and strictly increasing, therefore for $x \in[a, b]$ and $t \in[a, x],(g(x)-g(t))^{\frac{\alpha}{k}-1} \leq(g(x)-g(a))^{\frac{\alpha}{k}-1}, \alpha \geq k$. Also $g^{\prime}(x)>0$ hence $g^{\prime}(t)(g(x)-g(t))^{\frac{\alpha}{k}-1} \leq g^{\prime}(t)(g(x)-g(a))^{\frac{\alpha}{k}-1}$. From convexity of $f$, we have $f(t) \leq \frac{x-t}{x-a} f(a)+\frac{t-a}{x-a} f(x)$. From the last two inequalities one can has the following integral inequality

$$
\begin{aligned}
& \int_{a}^{x}(g(x)-g(t))^{\frac{\alpha}{k}-1} f(t) g^{\prime}(t) d t \\
& \leq \frac{(g(x)-g(a))^{\frac{\alpha}{k}-1}}{x-a}\left[f(a) \int_{a}^{x}(x-t) g^{\prime}(t) d t+f(x) \int_{a}^{x}(t-a) g^{\prime}(t) d t\right] .
\end{aligned}
$$

By using the Definition 4 , we get

$$
\begin{aligned}
& k \Gamma_{k}(\alpha) I_{g, a^{+}}^{\alpha, k} f(x) \\
& \leq \frac{\left((g(x)-g(a))^{\frac{\alpha}{k}-1}\right.}{x-a}\left[(x-a)(f(x) g(x)-f(a) g(a))-(f(x)-f(a)) \int_{a}^{x} g(t) d t\right] .
\end{aligned}
$$

Now on the other hand for $x \in[a, b], t \in[x, b], g^{\prime}(t)(g(x)-g(t))^{\frac{\beta}{k}-1} \leq g^{\prime}(t)(g(b)-$ $g(x))^{\frac{\beta}{k}-1}, \beta \geq k$. From convexity of $f$ we have $f(t) \leq \frac{t-x}{b-x} f(b)+\frac{b-t}{b-x} f(x)$. 
Multiplying the last two inequalities and integrating over $[x, b]$ one can has the integral inequality, which in addition with (6) constitutes the required estimate.

$$
\begin{aligned}
& k \Gamma_{k}(\beta) I_{g, b^{-}}^{\beta, k} f(x) \\
& \leq \frac{(g(b)-g(x))^{\frac{\beta}{k}-1}}{b-x}\left[(b-x)(f(b) g(b)-f(x) g(x))-(f(b)-f(x)) \int_{x}^{b} g(t) d t\right] .
\end{aligned}
$$

A special case of above theorem is stated in the following corollary which gives [4, Theorem 1] for $k=1, g$ as identity function.

Corollary 7. If the assumptions of Theorem 6 hold, then the following fractional integral inequality holds

$$
\begin{aligned}
& k \Gamma_{k}(\alpha)\left(I_{g, a^{+}}^{\alpha, k} f(x)+I_{g, b^{-}}^{\alpha, k} f(x)\right) \\
& \leq \frac{\left((g(x)-g(a))^{\frac{\alpha}{k}-1}\right.}{x-a}\left[(x-a)(f(x) g(x)-f(a) g(a))-(f(x)-f(a)) \int_{a}^{x} g(t) d t\right] \\
& +\frac{\left((g(b)-g(x))^{\frac{\alpha}{k}-1}\right.}{b-x}\left[(b-x)(f(b) g(b)-f(x) g(x))-(f(b)-f(x)) \int_{x}^{b} g(t) d t\right]
\end{aligned}
$$

Next theorem is the modulus fractional inequality that derives some known results.

Theorem 8. Let $f, g:[a, b] \longrightarrow \mathbb{R}$ be real valued functions with $a<b$. Also let $f$ be differentiable and $\left|f^{\prime}\right|$ is convex, and $g$ be also differentiable and strictly increasing with $g^{\prime} \in L[a, b]$. Then for $\alpha, \beta \geq 0$ and $k>0$, the following modulus fractional inequality holds

$$
\begin{aligned}
& \mid \Gamma_{k}(\alpha+k) I_{g, a^{+}}^{\alpha, k} f(x)+\Gamma_{k}(\beta+k) I_{g, b^{-}}^{\beta, k} f(x) \\
& -\left((g(x)-g(a))^{\frac{\alpha}{k}} f(a)+(g(b)-g(x))^{\frac{\beta}{k}} f(b)\right) \mid \\
& \leq \frac{(g(x)-g(a))^{\frac{\alpha}{k}}(x-a)\left|f^{\prime \frac{\beta}{k}}(b-x)\right| f^{\prime}(b) \mid}{2} \\
& +\left|f^{\prime}(x)\right| \frac{\left((g(x)-g(a))^{\frac{\alpha}{k}}(x-a)+(g(b)-g(x))^{\frac{\beta}{k}}(b-x)\right)}{2} .
\end{aligned}
$$

Proof. From convexity of $\left|f^{\prime}\right|$, we have $\left|f^{\prime}(t)\right| \leq \frac{x-t}{x-a}\left|f^{\prime}(a)\right|+\frac{t-a}{x-a}\left|f^{\prime}(x)\right|$ which gives $f^{\prime}(t) \leq \frac{x-t}{x-a}\left|f^{\prime}(a)\right|+\frac{t-a}{x-a}\left|f^{\prime}(x)\right|$. Since the function $g$ is differentiable and strictly increasing therefore we have $g(x)-g(t))^{\frac{\alpha}{k}} \leq(g(x)-g(a))^{\frac{\alpha}{k}}$, where as $x \in[a, b]$ and $t \in[a, x], \alpha \geq 0, k>0$.

The product of last two inequalities give

$$
(g(x)-g(t))^{\frac{\alpha}{k}} f^{\prime}(t) \leq \frac{(g(x)-g(a))^{\frac{\alpha}{k}}}{x-a}\left((x-t)\left|f^{\prime}(a)\right|+(t-a)\left|f^{\prime}(x)\right|\right) .
$$


Integrating with respect to $t$ over $[a, x]$, we have

$$
\begin{aligned}
& \int_{a}^{x}(g(x)-g(t))^{\frac{\alpha}{k}} f^{\prime}(t) d t \\
& \leq \frac{(g(x)-g(a))^{\frac{\alpha}{k}}}{x-a}\left[\left|f^{\prime}(a)\right| \int_{a}^{x}(x-t) d t+\left|f^{\prime}(x)\right| \int_{a}^{x}(t-a) d t\right] \\
& =(g(x)-g(a))^{\frac{\alpha}{k}}(x-a)\left[\frac{\left|f^{\prime}(a)\right|+\left|f^{\prime}(x)\right|}{2}\right]
\end{aligned}
$$

and

$$
\begin{aligned}
& \left.\int_{a}^{x}(g(x)-g(t))^{\frac{\alpha}{k}} f^{\prime \frac{\alpha}{k}}\right|_{a} ^{x}+\frac{\alpha}{k} \int_{a}^{x}(g(x)-g(t))^{\frac{\alpha}{k}-1} f(t) g^{\prime}(t) d t \\
= & -f(a)(g(x)-g(a))^{\frac{\alpha}{k}}+\Gamma_{k}(\alpha+k) I_{g, a^{+}}^{\alpha, k} f(x) .
\end{aligned}
$$

Therefore 9 takes the form

$\Gamma_{k}(\alpha+k) I_{g, a^{+}}^{\alpha, k} f(x)-f(a)(g(x)-g(a))^{\frac{\alpha}{k}} \leq(g(x)-g(a))^{\frac{\alpha}{k}}(x-a)\left[\frac{\left|f^{\prime}(a)\right|+\left|f^{\prime}(x)\right|}{2}\right]$.

Also from convexity of $\left|f^{\prime}\right|$ one can has $f(t) \geq-\left(\frac{x-t}{x-a}\left|f^{\prime}(a)\right|+\frac{t-a}{x-a}\left|f^{\prime}(x)\right|\right)$ and following the same procedure as we did to get 10 next inequality holds

$f(a)(g(x)-g(a))^{\frac{\alpha}{k}}-\Gamma_{k}(\alpha+k) I_{g, a^{+}}^{\alpha, k} f(x) \leq(g(x)-g(a))^{\frac{\alpha}{k}}(x-a)\left[\frac{\left|f^{\prime}(a)\right|+\left|f^{\prime}(x)\right|}{2}\right]$.

Inequalities 10 and 11) provide the modulus inequality

$$
\begin{aligned}
& \left|\Gamma_{k}(\alpha+k) I_{g, a^{+}}^{\alpha, k} f(x)-f(a)(g(x)-g(a))^{\frac{\alpha}{k}}\right| \\
& \leq(g(x)-g(a))^{\frac{\alpha}{k}}(x-a)\left[\frac{\left|f^{\prime}(a)\right|+\left|f^{\prime}(x)\right|}{2}\right] .
\end{aligned}
$$

On the other hand from convexity of $\left|f^{\prime}\right|$ we have $\left|f^{\prime}(t)\right| \leq \frac{t-x}{b-x}\left|f^{\prime}(b)\right|+\frac{b-t}{b-x}\left|f^{\prime}(x)\right|$, for $x \in[a, b]$ and $t \in[x, b]$ and $\beta \geq 0, k>0$. Also the inequality $(g(t)-g(x))^{\frac{\beta}{k}} \leq$ $(g(b)-g(x))^{\frac{\beta}{k}}$ holds true for function $g$. Following the same way as we have done to obtain 12 the following inequality holds

$$
\left|\Gamma_{k}(\beta+k) I_{g, b^{-}}^{\beta, k} f(x)-f(b)(g(b)-g(x))^{\frac{\beta}{k}}\right| \leq(g(b)-g(x))^{\frac{\beta}{k}}(b-x)\left[\frac{\left|f^{\prime}(b)\right|+\left|f^{\prime}(x)\right|}{2}\right] .
$$

From inequalities $(12)$ and $(13)$ via triangular inequality we get (8) which is required.

A special case of above theorem is stated in the following corollary which gives [4. Theorem 2] for $k=1, g$ as identity function. 
Corollary 9. If the assumptions of Theorem 8 hold, then the following fractional integral inequality holds

$$
\begin{aligned}
& \left|\Gamma_{k}(\alpha+k)\left(I_{g, a^{+}}^{\alpha, k} f(x)+I_{g, b^{-}}^{\alpha, k} f(x)\right)-\left((g(x)-g(a))^{\frac{\alpha}{k}} f(a)+(g(b)-g(x))^{\frac{\alpha}{k}} f(b)\right)\right| \\
& \leq \frac{(g(x)-g(a))^{\frac{\alpha}{k}}(x-a)\left|f^{\prime \frac{\alpha}{k}}(b-x)\right| f^{\prime}(b) \mid}{2} \\
& +\left|f^{\prime}(x)\right| \frac{\left((g(x)-g(a))^{\frac{\alpha}{k}}(x-a)+(g(b)-g(x))^{\frac{\alpha}{k}}(b-x)\right)}{2} .
\end{aligned}
$$

The following lemma is useful to prove the next result.

Lemma 10. 4] Let $f:[a, b] \longrightarrow \mathbb{R}$, be a convex function. If $f$ is symmetric about $\frac{a+b}{2}$, then the following inequality holds

$$
f\left(\frac{a+b}{2}\right) \leq f(x) \quad x \in[a, b]
$$

Theorem 11. Let $f, g:[a, b] \longrightarrow \mathbb{R}$ be real valued functions with $a<b$. Also let $f$ be positive convex and symmetric about $\frac{a+b}{2}$, and $g$ be differentiable and strictly increasing with $g^{\prime} \in L[a, b]$. Then for $\alpha, \beta \geq 0$ and $k>0$, we have the following fractional inequality

$$
\begin{aligned}
& f\left(\frac{a+b}{2}\right)\left[\Gamma_{k}(\alpha+k) I_{g, a^{+}}^{\alpha, k} g(b)-\Gamma_{k}(\beta+k) I_{g, b^{-}}^{\beta, k} g(a)\right. \\
& \left.-(g(b)-g(a))^{\frac{\alpha}{k}} g(a)+(g(b)-g(a))^{\frac{\beta}{k}} g(b)\right] \\
& \leq \Gamma_{k}(\alpha+1) I_{g, a^{+}}^{\alpha+1, k} f(b)+\Gamma_{k}(\beta+1) I_{g, b^{-}}^{\beta+1, k} f(a) \\
& \leq \frac{\left((g(b)-g(a))^{\frac{\beta}{k}}+(g(b)-g(a))^{\frac{\alpha}{k}}\right)}{b-a} \\
& \times\left[(b-a)(f(b) g(b)-f(a) g(a))-(f(b)-f(a)) \int_{a}^{b} g(x) d x\right] .
\end{aligned}
$$

Proof. Since the function $g$ is differentiable and strictly increasing therefore $(g(x)-$ $g(a))^{\frac{\beta}{k}} \leq(g(b)-g(a))^{\frac{\beta}{k}}$, where as $x \in[a, b], \beta \geq 0, k>0$. Also $g^{\prime}(x)>0$ hence the inequality $g^{\prime}(x)(g(x)-g(a))^{\frac{\beta}{k}} \leq g^{\prime}(x)(g(b)-g(a))^{\frac{\beta}{k}}$ holds true. From convexity of $f, f(x) \leq \frac{x-a}{b-a} f(b)+\frac{b-x}{b-a} f(a)$. The product of last two inequalities is integrated over $[a, b]$ to get

$$
\begin{aligned}
& \int_{a}^{b}(g(x)-g(a))^{\frac{\beta}{k}} f(x) g^{\prime}(x) d x \\
& \leq \frac{(g(b)-g(a))^{\frac{\beta}{k}}}{b-a}\left[f(b) \int_{a}^{b}(x-a) g^{\prime}(x) d x+f(a) \int_{a}^{b}(b-x) g^{\prime}(x) d x\right] .
\end{aligned}
$$


By using Definition (4) we get

$$
\begin{aligned}
& \Gamma_{k}(\beta+1) I_{g, b^{-}}^{\beta+1, k} f(a) \\
& \leq \frac{(g(b)-g(a))^{\frac{\beta}{k}}}{b-a}\left[(b-a)(f(b) g(b)-f(a) g(a))-(f(b)-f(a)) \int_{a}^{b} g(x) d x\right] .
\end{aligned}
$$

Now for $x \in[a, b], t \in[x, b]$ and $\alpha \geq 0, k>0$, the inequality $g^{\prime}(x)(g(b)-g(x))^{\frac{\beta}{k}} \leq$ $g^{\prime}(x)(g(b)-g(a))^{\frac{\beta}{k}}$ holds true. Following the same way as we have done to get 16 the following inequality can be obtained

$$
\begin{aligned}
& \Gamma_{k}(\alpha+1) I_{g, a^{+}}^{\alpha+1, k} f(b) \\
& \leq \frac{(g(b)-g(a))^{\frac{\alpha}{k}}}{b-a}\left[(b-a)(f(b) g(b)-f(a) g(a))-(f(b)-f(a)) \int_{a}^{b} g(x) d x\right] .
\end{aligned}
$$

From (16) and (17), we get

$$
\begin{aligned}
& \Gamma_{k}(\alpha+1) I_{g, a^{+}}^{\alpha+1, k} f(b)+\Gamma_{k}(\beta+1) I_{g, b^{-}}^{\beta+1, k} f(a) \\
& \leq \frac{\left((g(b)-g(a))^{\frac{\beta}{k}}+(g(b)-g(a))^{\frac{\alpha}{k}}\right)}{b-a} \\
& \times\left[(b-a)(f(b) g(b)-f(a) g(a))-(f(b)-f(a)) \int_{a}^{b} g(x) d x\right] .
\end{aligned}
$$

Using Lemma 10 and multiplying 14 with $(g(x)-g(a))^{\frac{\beta}{k}} g^{\prime}(x)$, then integrating over $[a, b]$, we have

$$
f\left(\frac{a+b}{2}\right) \int_{a}^{b}(g(x)-g(a))^{\frac{\beta}{k}} g^{\prime}(x) d x \leq \int_{a}^{b}(g(x)-g(a))^{\frac{\beta}{k}} g^{\prime}(x) f(x) d x .
$$

By using Definition 3 , we get

$$
f\left(\frac{a+b}{2}\right)\left[(g(b)-g(a))^{\frac{\beta}{k}} g(b)-\Gamma_{k}(\beta+k) I_{g, b^{-}}^{\beta, k} g(a)\right] \leq \Gamma_{k}(\beta+1) I_{g, b^{-}}^{\beta+1, k} f(a) .
$$

Similarly, using Lemma 10 and multiplying 14 with $(g(b)-g(x))^{\frac{\beta}{k}} g^{\prime}(x)$, then integrating over $[a, b]$, we have

$$
f\left(\frac{a+b}{2}\right)\left[\Gamma_{k}(\alpha+k) I_{g, a^{+}}^{\alpha, k} g(b)-(g(b)-g(a))^{\frac{\beta}{k}} g(a)\right] \leq \Gamma_{k}(\alpha+1) I_{g, a^{+}}^{\alpha+1, k} f(b) .
$$

From 20 and 21 the following inequality holds which with 180 constitute 15 .

$$
\begin{aligned}
& f\left(\frac{a+b}{2}\right)\left[\Gamma_{k}(\alpha+k) I_{g, a^{+}}^{\alpha, k} g(b)-\Gamma_{k}(\beta+k) I_{g, b^{-}}^{\beta, k} g(a)\right. \\
& \left.-(g(b)-g(a))^{\frac{\alpha}{k}} g(a)+(g(b)-g(a))^{\frac{\beta}{k}} g(b)\right]
\end{aligned}
$$




$$
\leq \Gamma_{k}(\alpha+1) I_{g, a^{+}}^{\alpha+1, k} f(b)+\Gamma_{k}(\beta+1) I_{g, b^{-}}^{\beta+1, k} f(a) .
$$

A special case of above theorem is stated in the following corollary which gives [4, Theorem 3] for $k=1, g$ as identity function.

Corollary 12. If the assumptions of Theorem 11 hold, then the following fractional integral inequality holds

$$
\begin{aligned}
& f\left(\frac{a+b}{2}\right)\left[\Gamma_{k}(\alpha+k)\left(I_{g, a^{+}}^{\alpha, k} g(b)-I_{g, b^{-}}^{\alpha, k} g(a)\right)\right. \\
& \left.-(g(b)-g(a))^{\frac{\alpha}{k}} g(a)+(g(b)-g(a))^{\frac{\alpha}{k}} g(b)\right] \\
& \leq \Gamma_{k}(\alpha+1)\left(I_{g, a^{+}}^{\alpha+1, k} f(b)+I_{g, b^{-}}^{\alpha+1, k} f(a)\right) \\
& \leq \frac{\left((g(b)-g(a))^{\frac{\alpha}{k}}+(g(b)-g(a))^{\frac{\alpha}{k}}\right)}{b-a} \\
& \times\left[(b-a)(f(b) g(b)-f(a) g(a))-(f(b)-f(a)) \int_{a}^{b} g(x) d x\right] .
\end{aligned}
$$

\section{Applichtions}

In this section we give applications of the results proved in the previous section. First we apply Theorem 6 and get the following result.

Theorem 13. Under the assumptions of Theorem 6, we have

$$
\begin{aligned}
& k \Gamma_{k}(\alpha) I_{g, a^{+}}^{\alpha, k} f(b)+k \Gamma_{k}(\beta) I_{g, b^{-}}^{\beta, k} f(a) \\
& \leq\left(\frac{(g(b)-g(a))^{\frac{\alpha}{k}-1}+(g(b)-g(a))^{\frac{\beta}{k}-1}}{b-a}\right) \\
& \times\left((b-a)(f(b) g(b)-f(a) g(a))-(f(b)-f(a)) \int_{a}^{b} g(t) d t\right) .
\end{aligned}
$$

Proof. If we take $x=a$ and $x=b$ in (5), then adding resulting inequalities, we get (22).

Corollary 14. If we take $\alpha=\beta$ in 22, then we have the following fractional integral inequality

$$
\begin{aligned}
& k \Gamma_{k}(\alpha)\left(I_{g, a^{+}}^{\alpha, k} f(b)+I_{g, b^{-}}^{\alpha, k} f(a)\right) \\
& \leq \frac{2(g(b)-g(a))^{\frac{\alpha}{k}-1}}{b-a}\left((b-a)(f(b) g(b)-f(a) g(a))-(f(b)-f(a)) \int_{a}^{b} g(t) d t\right) .
\end{aligned}
$$


Corollary 15. If we take $\alpha=k=1$ and $g(x)=x$ in 23), then we get the following inequality

$$
\frac{1}{b-a} \int_{a}^{b} f(t) d t \leq \frac{f(a)+f(b)}{2} .
$$

Next we apply Theorem 8 to obtain required results.

Theorem 16. Under the assumptions of Theorem 8, we have

$$
\begin{aligned}
& \mid \Gamma_{k}(\alpha+k) I_{g, a^{+}}^{\alpha, k} f\left(\frac{a+b}{2}\right)+\Gamma_{k}(\beta+k) I_{g, b^{-}}^{\beta,, k} f\left(\frac{a+b}{2}\right) \\
& -\left(\left(g\left(\frac{a+b}{2}\right)-g(a)\right)^{\frac{\alpha}{k}} f(a)+\left(g(b)-g\left(\frac{a+b}{2}\right)\right)^{\frac{\beta}{k}} f(b)\right) \mid \\
& \leq \frac{\left(\frac{b-a}{2}\right)\left(\left(g\left(\frac{a+b}{2}\right)-g(a)\right)^{\frac{\alpha}{k}}\left|f^{\prime}(a)\right|+\left(g(b)-g\left(\frac{a+b}{2}\right)\right)^{\frac{\beta}{k}}\left|f^{\prime}(b)\right|\right)}{2} \\
& +\left|f^{\prime}\left(\frac{a+b}{2}\right)\right| \frac{\left(\frac{b-a}{2}\right)\left(\left(g\left(\frac{a+b}{2}\right)-g(a)\right)^{\frac{\alpha}{k}}+\left(g(b)-g\left(\frac{a+b}{2}\right)\right)^{\frac{\beta}{k}}\right)}{2} .
\end{aligned}
$$

Proof. If we take $x=\frac{a+b}{2}$ in (8), then resulting inequality (25) can be obtained.

Corollary 17. If we take $\alpha=\beta$ in 25), then we have the following fractional integral inequality

$$
\begin{aligned}
& \mid \Gamma_{k}(\alpha+k)\left(I_{g, a^{+}}^{\alpha, k} f\left(\frac{a+b}{2}\right)+I_{b^{-}}^{\alpha, g} f\left(\frac{a+b}{2}\right)\right) \\
& -\left(\left(g\left(\frac{a+b}{2}\right)-g(a)\right)^{\frac{\alpha}{k}} f(a)+\left(g(b)-g\left(\frac{a+b}{2}\right)\right)^{\frac{\alpha}{k}} f(b)\right) \mid \\
& \leq \frac{\left(\frac{b-a}{2}\right)\left(\left(g\left(\frac{a+b}{2}\right)-g(a)\right)^{\frac{\alpha}{k}}\left|f^{\prime}(a)\right|+\left(g(b)-g\left(\frac{a+b}{2}\right)\right)^{\frac{\alpha}{k}}\left|f^{\prime}(b)\right|\right)}{2} \\
& +\left|f^{\prime}\left(\frac{a+b}{2}\right)\right| \frac{\left(\frac{b-a}{2}\right)\left(\left(g\left(\frac{a+b}{2}\right)-g(a)\right)^{\frac{\alpha}{k}}+\left(g(b)-g\left(\frac{a+b}{2}\right)\right)^{\frac{\alpha}{k}}\right)}{2} .
\end{aligned}
$$

Corollary 18. If we take $\alpha=k=1$ and $g(x)=x$ in (26), then we get the following inequality

$$
\left|\frac{1}{b-a} \int_{a}^{b} f(t) d t-\frac{f(a)+f(b)}{2}\right| \leq \frac{b-a}{8}\left[\left|f^{\prime}(a)\right|+\left|f^{\prime}(b)\right|+2\left|f^{\prime}\left(\frac{a+b}{2}\right)\right|\right] .
$$

It is interesting to note that for $\left.f^{\prime}\left(\frac{a+b}{2}\right)=0 \sqrt{27}\right)$ produce [3, Theorem 2.2]. If $f^{\prime}(x) \leq 0$, then (27) provides the refinement of [3, Theorem 2.2]. 


\section{Concluding Remarks}

This paper gives estimates of (RL) fractional integral in general form by means of convex functions. These estimates provide the estimations of (RL) and ( $k \mathrm{RL})$ fractional integrals and also for all fractional integrals comprises in Remark 1. Some related fractional inequalities are also obtained for differentiable functions having convex derivatives in absolute value. Applications of Theorem 6 and Theorem 8 are given by connecting some known results. By applying Theorem 11 similar results can be established which are left for the reader.

Acknowledgment. This research work is supported by Higher Education Commission of Pakistan under NRPU 2016, Project No. 5421.

\section{REFERENCES}

[1] Chen, H. and Katugampola, U. N., Hermite-Hadamard and Hermite-Hadamard-Fejér type inequalities for generalized fractional integrals, J. Math. Anal. Appl., 446 (2017), 1274-1291.

[2] Diaza, R. and Pariglan, E., On hypergeometric functions and Pochhammer $k$-symbol, Divulg. Mat., 15(2) (2007), 179-192.

[3] Dragomir, S. S., Agarwal, R. P., Two inequalities for differentiable mappings and applications to special means of real numbers and to trapezoidal formula, Appl. Math. Lett., 11(5) (1998), 91-95.

[4] Farid, G., Some Riemann-Liouville fractional integral inequalities for convex functions, $J$. Anal., (2018), doi.org/10.1007/s41478-0079-4.

[5] Farid, G., Rehman, A. U., and Zahra, M., On Hadamard inequalities for $k$-fractional integrals, Nonlinear Funct. Anal. Appl., 21(3) (2016), 463-478.

[6] Habib, S., Mubeen, S., and Naeem, M. N., Chebyshev type integral inequalities for generalized $k$-fractional conformable integrals, J. Inequal. Spec. Funct., 9(4) (2018), 53-65.

[7] Jarad, F., Ugurlu, E., Abdeljawad, T., and Baleanu, D., On a new class of fractional operators, Adv. Difference Equ., (2017), 2017:247.

[8] Khan, T. U., and Khan, M. A., Generalized conformable fractional operators, J. Comput. Appl. Math., 346 (2019), 378-389.

[9] Kilbas, A. A., Srivastava, H. M., and Trujillo, J. J., Theory and applications of fractional differential equations, North-Holland Mathematics Studies, 204, Elsevier, New York-London, 2006.

[10] Kwun, Y. C., Farid, G., Nazeer, W., Ullah, S., Kang, S. M., Generalized Riemann-Liouville $k$ fractional integrals associated with Ostrowski type inequalities and error bounds of Hadamard inequalities, IEEE Access, 6 (2018), 64946-64953.

[11] Lazarević, M., Advanced topics on applications of fractional calculus on control problems, System stability and modeling, WSEAS Press, 2014.

[12] Letnikov, A. V., Theory of differentiation with an arbitrary index (Russian), Moscow, Matem. Sbornik, 3 (1868), 1-66.

[13] Samko, S. G., Kilbas, A. A., and Marichev, O. I., Fractional integrals and derivatives: Theory and Applications, Gordon and Breach Science Publishers, 1993.

[14] Roberts, A. W., Varberg, D. E., Convex functions, Academic Press, New York, 1973.

[15] Sabatier, J., Agrawal, O. P., and J. T. Machado, Advances in fractional calculus: Theoretical developments and applications in Physics and Engineering, Springer 2007.

[16] Sarikaya, M. Z., Dahmani, M., Kiris, M. E., and Ahmad, F., $(k, s)$-Riemann-Liouville fractional integral and applications, Hacet. J. Math. Stat., 45(1) (2016), 77-89.

[17] Sonin, N. Y., On differentiation with arbitray index, Moscow Matem. Sbornik, 6(1) (1869), $1-38$. 
Current address: Ghulam Farid: COMSATS University Islamabad, Attock Campus, Pakistan.

E-mail address: faridphdsms@hotmail.com, ghlmfarid@cuiatk.edu.pk

ORCID Address: https://orcid.org/0000-0002-4103-7745 